\title{
Caracterización Estereológica de Odontoblastos en Pulpas Dentarias Humanas Sanas y con Pulpitis Reversible
}

\author{
Stereological Characterization of Odontoblasts in Normal Healthy \\ and Reversible Pulpitis in Human Dental Pulps
}

Rolando Morales*; Erick Trujillo** \& Mario Cantín ${ }^{* * *, * * * * *}$

MORALES, R.; TRUJILLO, E. \& CANTÍN, M. Caracterización estereológica de odontoblastos en pulpas dentarias humanas sanas y con pulpitis reversible. Int. J. Morphol., 32(1):154-160, 2014.

RESUMEN: La reacción y reparación dentinaria depende del número de odontoblastos. Los métodos para obtener estimaciones fiables sobre la cantidad de odontoblastos en la pulpa dental han sido subjetivos y sesgados, sobre todo al evaluar los cambios cuantitativos y la potencial capacidad reparativa en presencia de caries. El objetivo de este trabajo fue estimar y comparar cuantitativamente el número, densidad y volumen de odontoblastos en dientes sanos y con diagnóstico de pulpitis reversible producto de caries a través de herramientas estereológicas. Se utilizaron dientes premolares humanos obtenidos de exodoncias, divididos en un grupo sano y otro cariado. Fueron fijados y descalcificados con ácido nítrico al 5\%. Siguiendo el protocolo del orientator se obtuvieron 5 secciones de 5 $\mathrm{mm}$ teñidas por H-E de cada diente. Se aplicó el recuento estereológico de los odontoblastos con el test multipropósito M42. Se estimaron las densidades de número $(\mathrm{Nv})$, volumen $(\mathrm{Vv})$ y superficie $(\mathrm{Sv})$, y calcularon las Medias $( \pm \mathrm{DE})$ por diente, y Medias $( \pm \mathrm{EE})$ por grupo. Las diferencias entre grupos se analizaron mediante la prueba $\mathrm{T}$, con un valor $\mathrm{p} \leq 0,05$ de significación estadística. En dientes sanos, la Media ( \pm EE) para Nv de odontoblastos fue $0,409 \times 105 / \mathrm{mm}^{3}\left( \pm 0,018 \times 10^{5} / \mathrm{mm}^{3}\right)$, para Vv $19,714 \%( \pm 1,43 \%)$ y para Sv 21,016 $\mathrm{mm}^{2} / \mathrm{mm}^{3}\left( \pm 1,425 \mathrm{~mm}^{2} / \mathrm{mm}^{3}\right)$. En dientes cariados, la Nv fue $0,521 \times 105 / \mathrm{mm} 3\left( \pm 0,023 \times 10^{5} / \mathrm{mm}^{3}\right)$, la Vv $24,686 \%( \pm 1,625 \%)$ y la Sv $23,203 \mathrm{~mm}^{2} / \mathrm{mm}^{3}\left( \pm 1,364 \mathrm{~mm}^{2} / \mathrm{mm}^{3}\right)$. Al comparar las $\mathrm{Nv}$, los odontoblastos del grupo con caries aumentaron significativamente ( $\mathrm{p}=0,0062)$, al igual que la $\mathrm{Vv}(\mathrm{p}=0,0197)$. Existe un aumento del número de odontoblastos en los dientes con pulpitis reversible, lo que condicionaría su capacidad de respuesta. La metodología empleada puede ser aplicable para determinar el comportamiento pulpar y cuantificar variables de respuesta odontoblástica en tratamientos restauradores atraumáticos de manera imparcial y reproducible.

PALABRAS CLAVE: Odontoblasto; Diente humano; Estereología; Pulpa dentaria.

\section{INTRODUCCIÓN}

La pulpa es el órgano que provee al diente de vitalidad mediante componentes vasculares y nerviosos, y mantiene su fisiología normal. También se encarga de la producción de dentina o dentinogénesis, proceso dinámico que ocurre tanto en etapas de maduración como ante la reacción a injurias o estímulos, ya sean traumáticos o infecciosos como la caries (Bruno et al., 2010).

Los odontoblastos son células altamente diferenciadas responsables de regular la síntesis, secreción y mineralización de la dentina durante la vida (Couble et al., 2000); estas han demostrado detectar y responder ante una injuria que afecta a la dentina incrementando su espesor, y forma una barrera entre los odontoblastos y el estímulo, función que depende de la capa odontoblástica (Brännström \& Lind, 1965; Bjorndal \& Darvann, 1999; Murray et al., 2001). La reacción y reparación dentinaria depende de diversos parámetros, incluyendo el número de odontoblastos (Murray et al., 2000; About et al., 2001). Otros fenotipos celulares de la pulpa dentaria, ubicados en la capa subodontoblástica y región central soportan a la dentinogénesis, pero no juegan un rol directo en la secreción de matriz dentinaria (Linde, 1985; Tziafas, 2010). Esto se debe a que nuevos odontoblastos se originan desde estos fenotipos celulares pulpares mediante procesos de diferenciación (Fitzgerald et al., 1990), aunque esta teoría es aún controversial (Tziafas, 1994).

\footnotetext{
* Cirujano Dentista, Servicio de Salud de Viña del Mar, Chile.

** Departamento de Ciencias Básicas Biomédicas, Universidad de Talca, Talca, Chile.

*** Programa de Doctorado en Ciencias Morfológicas; Facultad de Odontología, Universidad de La Frontera, Temuco, Chile.

**** Centro de Investigación en Ciencias Biomédicas, Universidad Autónoma de Chile, Temuco, Chile.
} 
La pulpa dentaria reacciona frente a los estímulos o injurias con dolor, inflamación y aposición de tejido dentinario (según la gravedad de la lesión y situación pulpar), en un cuadro definido como pulpitis (Trowbridge et al., 2002). A partir de esta evidencia, se realiza un diagnóstico clínico e histológico. El primero se basa en la historia del paciente y diente afectado, sus signos, síntomas y el resultado de pruebas diagnóstica, el cual no se condice necesariamente con el diagnóstico histológico (Pashley et al., 2002; Murray et al., 2002).

El diagnóstico clínico especula en la posibilidad de la pulpa de repararse a sí misma si desaparece o se controla el estímulo, estado definido como pulpitis reversible. En estos casos, histológicamente se han descrito cambios tisulares y características histomorfológicas propias (Vermelin et al., 1995; Goldberg et al., 2008; Vavpotic et al., 2009). Sin embargo, este método evalúa principalmente la reacción del tejido pulpar ante una lesión de manera cualitativa, lo cual es subjetivo (Warfvinge, 1987).

Cambios cuantificables en la pulpa dental no están descritos a cabalidad, y aún no se ha estimado la cantidad y densidad de odontoblastos en un estado de salud o reacción pulpar (Goldberg et al.). Hasta hace poco, los métodos para obtener estimaciones fiables sobre la cantidad de odontoblastos en la pulpa dental han sido insuficientes. La aplicación de herramientas estereológicas constituyen un método fiable para estimar la cantidad de células en un tejido biológico determinado (Geinisman et al., 1996; Mandarim-de-Lacerda, 2003; Sentut et al., 2012).

El objetivo fue estimar y comparar cuantitativamente el número, densidad y volumen de odontoblastos en pulpas sanas y con diagnóstico clínico de pulpitis reversible producto de caries en dientes humanos jóvenes.

\section{MATERIAL Y MÉTODO}

Se realizó un estudio de corte tranversal. La muestra fue seleccionada mediante un muestreo aleatorio simple, correspondiente a dientes premolares humanos, clínicamente sanos o con diagnóstico pulpitis reversible producida por caries, obtenidos de exodoncias practicadas en servicios públicos de la Séptima Región y la Clínica de Urgencias e Internado de Cirugía de la Escuela de Odontología, Universidad de Talca, entre Marzo y Agosto del año 2012. Fueron incluidos dientes con indicación de exodoncia por necesidad de tratamiento ortodóntico, de pacientes con edades entre 15 y 20 años. Se excluyeron dientes con atrición evidente, restauraciones de cualquier tipo o extensión, o cuya exodoncia implicara osteotomía u odontosección. Los participantes menores de 15 años debieron dar su asentimiento junto a la firma del consentimiento de su apoderado o responsable legal, y los mayores de 18 años firmaron el consentimiento para ser parte del estudio. El protocolo de investigación fue revisado y aprobado por el Comité de Ética Científica de la Universidad de Talca (00184).

La muestra se constituyó por 10 dientes, divididos en 2 grupos de manera equilibrada: control (dientes sanos sin caries) $(\mathrm{n}=5)$ y cariados (dientes con diagnóstico de pulpitis reversible) $(\mathrm{n}=5)$. Inmediatos a su exodoncia, los dientes fueron fijados con formalina tamponada al $10 \%$ por 24 horas, y luego se sometieron a inmersión en ácido nítrico al 5\% para su descalcificación durante 14 días (Bränsström \& Lind). Posteriormente, fueron deshidratados en alcohol graduado y se embebieron in toto en parafina utilizando una procesadora de tejidos Microm ${ }^{\circledR}$ modelo STP 120. Los dientes se orientaron en los bloques de parafina de modo que las se pudieran cortar y obtener secciones mesio-distales paralelas a su eje longitudinal.

Cada diente se cortó a la mitad, exponiendo al tejido pulpar hacia una de las caras libre del taco para facilitar su corte, siguiendo el protocolo del orientator (Mattfeldt et al., 1990). Se obtuvieron 5 cortes de cada diente, con un grosor de $5 \mathrm{~mm}$ para ser teñidos mediante H-E. Se confirmó histológicamente el diagnóstico de pulpitis reversible. En las muestras de ambos grupos, se identificó la capa odontoblástica y analizaron 5 campos por cada corte, totalizando 125 campos por diente. Las muestras fueron observadas en un microscopio óptico Carl Zeiss® Primo Star con una cámara Canon ${ }^{\circledR}$ modelo EOS Rebel Xsi integrada. Las imágenes obtenidas se proyectaron en un monitor Samsung ${ }^{\circledR}$ HD, y se aplicó el recuento estereológico con el test multipropósito M42 (Mandarim de-la-Cerda, 2003).

Los parámetros estereológicos estimados fueron densidad de número $(\mathrm{Nv})$, densidad de volumen $(\mathrm{Vv})$ y densidad de superficie (Sv). Se calculó la Media y desviación estándar para cada diente, y la Media y error estándar para cada grupo. En todos los casos se utilizó un intervalo de confianza de $95 \%$. Las diferencias entre los grupos se analizaron mediante la prueba $\mathrm{T}$ para variables paramétricas independientes. Los valores de Medias se consideraron estadísticamente significativos con un valor $p \leq 0,05$. El análisis estadístico se realizó con el programa STATA versión 11.0 y los datos fueron graficados con el programa GraphPad Prism 5. El estudio fue financiado por el Departamento de Morfología de la Universidad de Talca, Chile. 


\section{RESULTADOS}

En el grupo control, la Nv Media de odontoblastos en el tejido pulpar fue $0,409 \times 105 / \mathrm{mm}^{3}$, con un error estándar de $\pm 0,018 \times 105 / \mathrm{mm}^{3}$. La Vv Media de odontoblastos fue $19,714 \%$ con un error estándar de $\pm 1,43 \%$. La Media del Sv de odontoblastos fue $21,016 \mathrm{~mm}^{2} / \mathrm{mm}^{3}$ con un error estándar de $\pm 1,425 \mathrm{~mm} 2 / \mathrm{mm}^{3}$. El detalle de la Media $( \pm \mathrm{DE})$ para las Nv, Vv y Sv de los odontoblastos de cada diente sano se observa en la Figura 1 (A-C).

En el grupo caries, la Nv Media de odontoblastos del tejido pulpar fue $0,521 \times 105 / \mathrm{mm}^{3}$, con un error estándar de $\pm 0,023 \times 105 / \mathrm{mm}^{3}$. La Vv Media fue $24,686 \%$ con un error estándar de $\pm 1,625 \%$. La Media del $\mathrm{Sv}$ fue 23,203 $\mathrm{mm}^{2} / \mathrm{mm}^{3}$ con un error estándar de $\pm 1,364 \mathrm{~mm}^{2} / \mathrm{mm}^{3}$. El detalle de la Media ( \pm DE) para las Nv, Vv y Sv de los odontoblastos de cada diente con caries se observa en la Figura 1 (D-F).

Al comparar la Nv de odontoblastos, en el grupo de dientes con caries el aumento observado fue significativo $(\mathrm{p}=0,0062)$ con respecto al grupo sano, al igual que lo observado al comparar la Vv ( $\mathrm{p}=0,0197)$. Se observaron diferencias entre los grupos con respecto a la Sv, pero no fueron significativas $(\mathrm{p}=0,1319)$. Las Medias, mínimos y máximos para cada parámetro analizado de los grupos y su comparación se observan en la Figura 2.
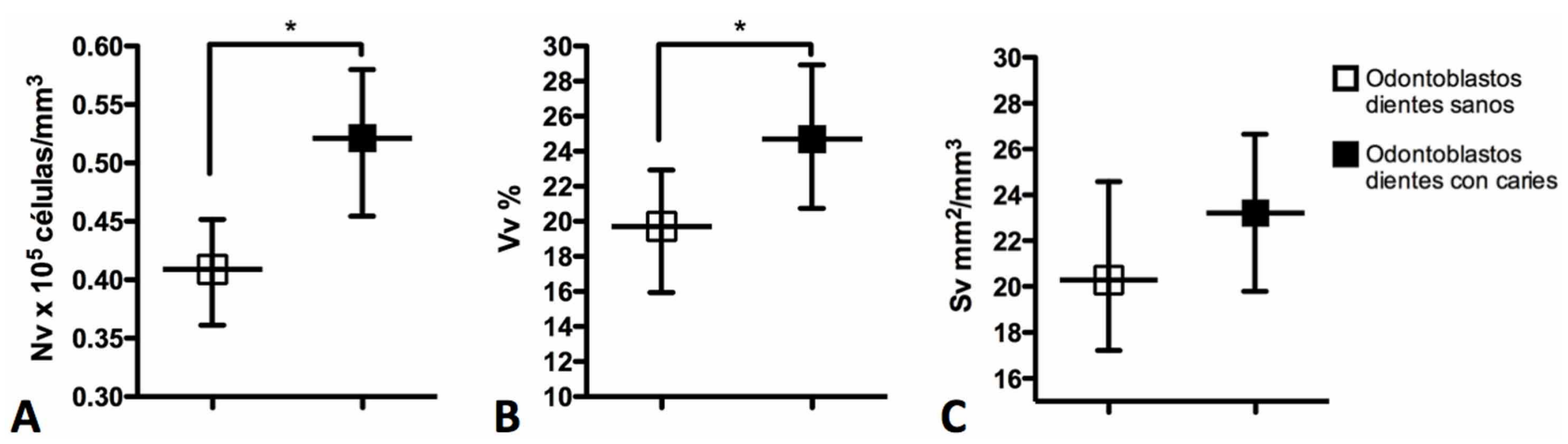

Fig. 1. Detalle de Media y DE para las variables Nv, Vv y Sv de los odontoblastos de cada diente del grupo sano (A, B y C) y con caries (D, E y F).

\section{DISCUSIÓN}

$\mathrm{Al}$ analizar los parámetros estereológicos $\mathrm{Nv}, \mathrm{Vv}$ y Sv de odontoblastos en dientes sanos y con caries, observamos un aumento de todas las densidades en presencia de caries. El aumento de la estimación del número de odontoblastos en dientes con caries en comparación a los sanos por mm3 fue significativa, lo que se refleja en la $\mathrm{Vv}$, con un incremento estimado significativo de su porcentaje desde $19,71 \%$ a $24,68 \%$, junto al aumento de la superficie ocupada por los odontoblastos en presencia de caries, sin embargo este último no fue significativo. Estos resultados difieren de los cambios iniciales descritos a nivel pulpar de un diente expuesto a caries u otra injuria; la reducción del número de odontoblastos, e incluso su tamaño, debido al incremento en la permeabilidad del esmalte y flujo de sustancias hacia el exterior e interior del diente, cambios hidrodinámicos que producen la aspiración y disgregación de los odontoblastos (Bränsström \& Lind; Vitalariu et al., 2005), lo que demuestra el potencial de reparación pulpar en los dientes analizados.

El aumento en el número y volumen de odontoblastos se puede explicar por el reclutamiento de células provenientes las capas más profundas de la pulpa dental, y no a un proceso de mitosis de los odontoblástos, ya que son células postmitóticas sin capacidad de dividirse (Arana-Chavez \& Massa, 2004); estas células se diferencian hacia odontoblastos y refuerzan la capa odontoblástica para reaccionar frente a la injuria. Corresponderían a células mesenquimáticas de la capa subodontoblástica, que responden a moléculas bioactivas y son objeto de divisiones, quimiotaxis, migraciones, adhesión y citodiferenciación celular, facultándolas para producir dentina (Arana-Chavez 

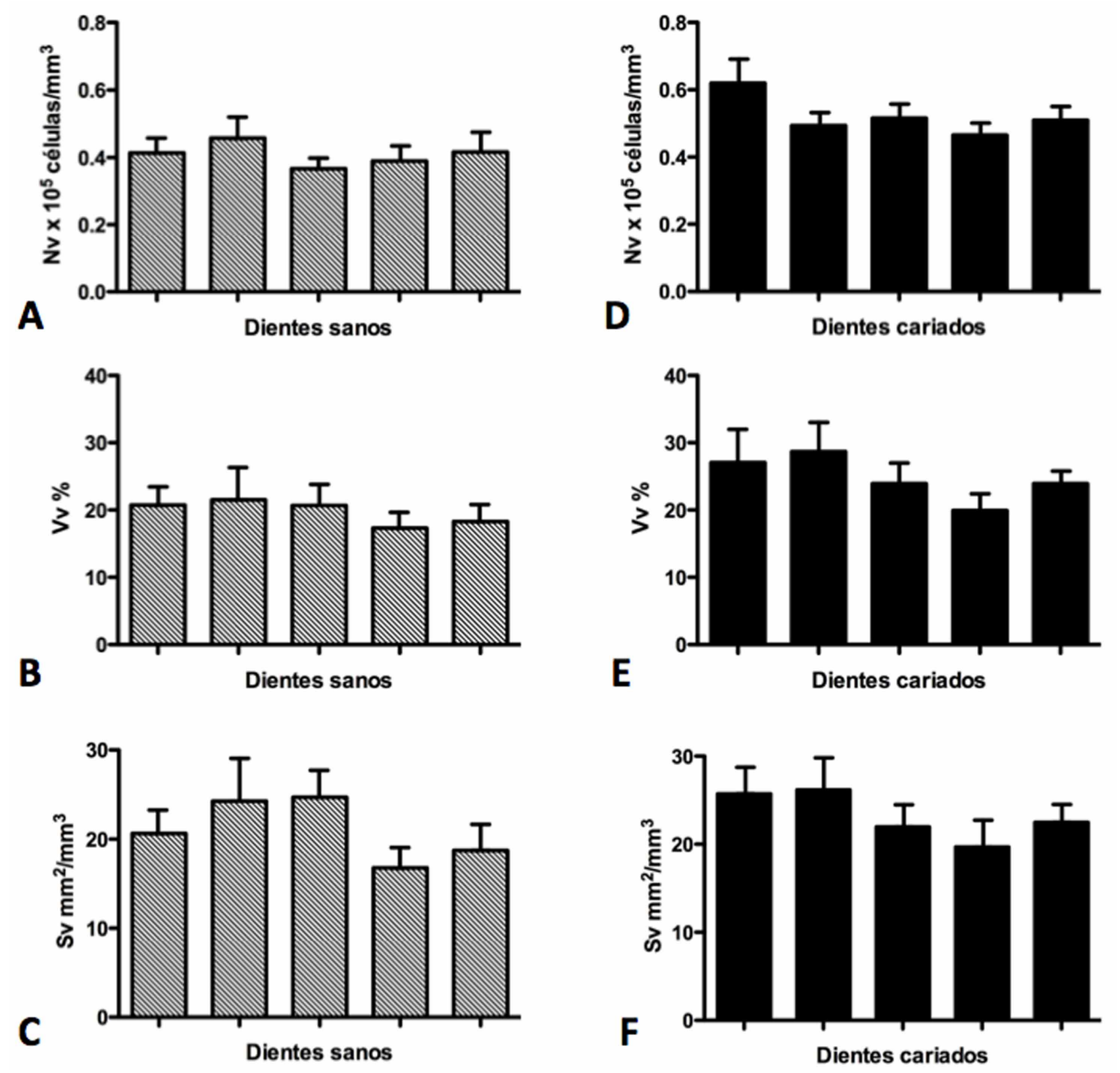

Fig. 2. Comparación de las variables Nv (A), Vv (B) y Sv (C) de odontoblastos de la capa odontoblástica entre los grupos de dientes sanos y con caries. $*=p \leq 0,05$.

\& Massa; Eslaminejad et al., 2010), transformándose en odontoblastos o bien una célula similar al odontoblastos, constituyendo una población progenitora importante y responsable del reemplazo de odontoblastos dañados, capaces de producir una matriz extracelular para generar tejido mineralizado (Murray et al., 2000; Goldberg et al.).

La compensación y aumento en el número de odontoblastos mediada por la aparición de un frente de rápida proliferación celular en la capa subodontoblástica
(Bjorndal \& Darvann), forma una monocapa de numerosas células ante la exposición a condiciones adversas como injurias y productos bacterianos, así como también en condiciones experimentales (Eslaminejad et al.). Por otra parte, frente al ataque de caries, moléculas MAP kinasas también inducen al aumento cuantitativo de fibroblastos (Gruber $e t$ al., 2004), sobretodo durante la inflamación pulpar. Junto a la masiva migración de células inflamatorias, mediadores de la inflamación como prostaglandinas y leucotrienos favorecen una reorientación del fenotipo celular y promueven 
la transdiferenciación de algunas células inflamatorias a células similares a odontoblastos, al igual que algunos fibroblastos son capaces de desarrollar este potencial después de su multiplicación (Goldberg et al.).

Los cambios celulares y estructurales de la pulpa dental frente al ataque de caries se relacionan directamente con su extensión y avance (Vitalariu et al.), y sería posible en una condición de pulpitis reversible como la analizada, con una lesión cariosa dentinaria de baja latencia, lo que permite una adecuada respuesta, diferenciación y proliferación de otros fenotipos celulares de la pulpa con potencial hacia células de tipo odontoblasto. La reactividad y capacidad de reparación frente a una injuria, en nuestro caso biológica, no sólo dependerá del número de odontoblastos (About et al.), sino que de otros factores tales como el espesor de la dentina, presencia de bacterias, de un material de restauración y su toxicidad, y la inflamación pulpar, como respuesta inmediata o acumulativa (Sentut et al., 2012).

El método más utilizado para evaluar las reacciones del tejido pulpar y capa odontoblástica es de tipo cualitativo, el cual presenta diversos elementos subjetivos y sesgos para analizar cambios en las estructuras biológicas (Warfvinge). Para cuantificar las estructuras morfológicas de una manera imparcial, reproducible y uniforme, el muestreo aleatorio y uso de herramientas estereológicas adecuadas son necesarias; además de aplicables con poco esfuerzo, rapidez (aunque consumen más tiempo que técnicas convencionales) y bajo costo. El uso de los principios estereológicos puede contribuir a eliminar de la controversia relacionada con la pérdida de odontoblastos con la edad (Murray et al., 2002), o cualquier otro proceso en el que la cuantificación de estructuras dinámicas puede ser de interés (Trujillo et al., 2011; Salgado et al., 2011); estos principios también han sido aplicados a otros elementos de la pulpa dentaria como los vasos sanguíneos; Santamaria et al. (2006), reportaron cambios de su $\mathrm{Vv}$ en dientes de ratas sometidas a movimientos ortodónticos, demostrando la alta capacidad de adaptación del tejido pulpar ante una agresión, siempre que los límites biológicos de la tolerancia de la pulpa se respeten.

Pocos estudios han cuantificado el número de odontoblastos. Murray et al. (2000), estimaron una densidad de 11.000 odontoblastos por $\mathrm{mm} 2$, lo cual se asemeja a lo reportado por Vavpotic et al., quienes estimaron una densidad promedio de 11.764 odontoblastos por $\mathrm{mm} 2$. Sin embargo, estas estimaciones fueron realizadas mediante histomorfometría bidimensional. Sentut et al. determinaron mediante métodos estereológicos el número de odontoblastos en dientes molares de niños entre 9 y 11 años expuestos y no a fluor. El número promedio de odontoblastos en segun- dos molares maxilares expuestos fue 630.775 , mientras que en los no expuestos fue de 665.200, en mandibulares expuesto 630.758 y en no expuestos 679.726. En el caso de los primeros molares maxilares el número en expuestos fue 370.203 y en no expuestos 399.594 , mientras que en mandibulares expuestos fue 522.077 y en no expuestos 534.534. Sin embargo, estos resultados no son comparables a los nuestros debido a la técnica de estimación utilizada.

Una limitación en nuestro estudio corresponde al tipo de diente utilizado, ya que según la serie analizada existen características diferenciales como el tamaño y superficie de la cámara pulpar que será ocupada por la pulpa y odontoblastos, así como el espesor del esmalte y dentina o configuración de sus prismas y túbulos, que influirán en el avance de la caries y respuesta pulpar. Por otra parte, el número de dientes analizados podría interpretarse como poco significativo, sin embargo esto es superado al utilizar el método estereológico, donde una de sus principales característica es la eficiencia de las muestras necesarias para el análisis, lo que significa el menor recuento posible con una alta precisión (Mandarim-de-Lacerda et al., 2010).

Los resultados obtenidos demuestran la capacidad de respuesta reparativa pulpar, pero cuando los límites biológicos de tolerancia son sobrepasados, y no es posible un aumento cuantitativo, entre otros, de los elementos celulares de la capa odontoblástica frente a una injuria debido a una lenta respuesta o estrés pulpar, bajo número de células potencialmente diferenciables hacia fenotipos odontoblásticos o muerte de los odontoblastos, la invasión bacteriana o la injuria pulpar desencadenará un proceso de pulpitis irreversible, necrosis, infección del sistema de canales radiculares y finalmente un proceso periapical (Goldberg et al.).

\section{CONCLUSIÓN}

En presencia de pulpitis reversible producto de caries, existe un aumento del número de odontoblastos en el tejido pulpar, el que condicionaría su capacidad de respuesta reparativa y la formación de dentina reaccional. La estimación cuantificable del número de odontoblastos nos entrega información sobre la capacidad reparativa de la pulpa expuesta a una injuria. Nuestros resultados y metodología empleada pueden ser aplicables para determinar el comportamiento pulpar y cuantificar variables de respuesta odontoblástica en procedimientos como el tratamiento restaurador atraumático u otros basados en la permanencia de dentina afectada por caries, y así evaluar su efectividad en la respuesta pulpar a través de parámetros cuantificables de manera imparcial y reproducible. 
MORALES, R.; TRUJILlo, E. \& CANTÍN, M. Stereological characterization of odontoblasts in normal healthy and reversible pulpitis human dental pulps. Int. J. Morphol., 32(1):154-160, 2014.

SUMMARY: Dentinal reaction and repair depends on factors like the amount of odontoblasts. Methods for obtaining reliable estimates of the number of odontoblasts in the dental pulp have been subjective and biased, especially when assessing the potential quantitative changes and reparative capacity in the presence of cavities. The aim of this study was to estimate and quantitatively compare, through stereological tools, the number, density and volume of odontoblasts in healthy teeth, diagnosed with reversible pulpitis due to caries. We used human premolars obtained from extractions, divided into groups of healthy teeth and teeth with caries, fixed and decalcified in $5 \%$ nitric acid. Following the orientator protocol, five 5 - $\mu \mathrm{m}$-thick sections stained by HE were obtained from each tooth. Stereological counting for odontoblast with M42 multipurpose test was applied. Numerical density (Nv), volume density (Vv) and surface density (Sv) were estimated, and the mean $( \pm \mathrm{SD})$ for each tooth, and Mean $( \pm \mathrm{SE})$ per group were calculated. Differences between groups were analyzed by $t$ test, with $p \leq 0.05$ for statistical significance. In the healthy teeth group, the mean ( \pm SE) for Nv was $0.409 \times 10^{5} / \mathrm{mm}^{3}\left( \pm 0.018 \times 10^{5} / \mathrm{mm}^{3}\right), \mathrm{Vv} 19.714 \%( \pm 1.43 \%)$ and to Sv $21.016 \mathrm{~mm}^{2} / \mathrm{mm}^{3}\left( \pm 1.425 \mathrm{~mm}^{2} / \mathrm{mm}^{3}\right)$ odontoblast cells. In the caries teeth group, the $\mathrm{Nv}$ was $0.521 \times 10^{5} / \mathrm{mm}^{3}\left( \pm 0.023 \times 10^{5} / \mathrm{mm}^{3}\right), \mathrm{Vv} 24.686 \%( \pm 1.625 \%)$ and $\mathrm{Sv} 23.203 \mathrm{~mm}^{2} / \mathrm{mm}^{3}\left( \pm 1.364 \mathrm{~mm}^{2} / \mathrm{mm}^{3}\right)$. When comparing Nv, an increased in odontoblasts significantly $(\mathrm{p}=0.0062)$, as well as $\mathrm{Vv}(\mathrm{p}=0.0197)$ in caries teeth group. There is an increased number of odontoblasts in teeth with reversible pulpitis, which would condition its responsiveness. The methodology can be applied to determine pulp behavior, and quantify variables of odontoblastic response in atraumatic restorative treatments in an impartial and reproducible form.

KEY WORDS: Odontoblast; Human tooth; Stereology; Dental pulp.

\section{REFERENCIAS BIBLIOGRÁFICAS}

About, I.; Murray, P. E.; Franquin, J. C.; Remusat, M. \& Smith, A. $\mathrm{J}$. The effect of cavity restoration variables on odontoblast cell numbers and dental repair. J. Dent., 29(2):109-17, 2001.

Arana-Chavez, V. E. \& Massa, L. F. Odontoblasts: the cells forming and maintaining dentine. Int. J. Biochem. Cell Biol., 36(8):1367-73, 2004.

Bjorndal, L. \& Darvann, T. A light microscopic study of odontoblastic and non-odontoblastic cells involved in tertiary dentinogenesis in well-defined cavitated carious lesions. $\mathrm{Ca}$ ries Res., 33(1):50-60, 1999.

Brännström, M. \& Lind, P. O. Pulpal response to early dental caries. J. Dent. Res., 44(5):1045-50, 1965.

Bruno, K. F.; Silva, J. A.; Silva, T. A.; Batista, A. C.; Alencar, A. H. \& Estrela, C. Characterization of inflammatory cell infiltrate in human dental pulpitis. Int. Endod. J., 43(11):1013-21, 2010.

Couble, M. L.; Farges, J. C.; Bleicher, F.; Perrat-Mabillon, B.; Boudeulle, M. \& Magloire, H. Odontoblast differentiation of human dental pulp cells in explant cultures. Calcif. Tissue Int., 66(2):129-38, 2000.

Eslaminejad, M. B.; Vahabi, S.; Shariati, M. \& Nazarian, H. In vitro Growth and Characterization of Stem Cells from Human Dental Pulp of Deciduous Versus Permanent Teeth. J. Dent. (Tehran), 7(4):185-95, 2010.

Fitzgerald, M.; Chiego, D. J. Jr. \& Heys, D. R. Autoradiographic analysis of odontoblast replacement following pulp exposure in primate teeth. Arch. Oral Biol., 35(9):707-15, 1990.
Geinisman, Y.; Gundersen, H. J.; van der Zee, E. \& West, M. J. Unbiased stereological estimation of the total number of synapses in a brain region. J. Neurocytol., 25(12):805-19, 1996.

Goldberg, M.; Farges, J. C.; Lacerda-Pinheiro, S.; Six, N.; Jegat, N.; Decup, F.; Septier, D.; Carrouel, F.; Durand, S.; ChaussainMiller, C.; Denbesten, P.; Veis, A. \& Poliard, A. Inflammatory and immunological aspects of dental pulp repair. Pharmacol. Res., 58(2):137-47, 2008.

Gruber, R.; Jindra, C.; Kandler, B.; Watzak, G.; Fischer, M. B. \& Watzek, G. Proliferation of dental pulp fibroblasts in response to thrombin involves mitogen-activated protein kinase signaling. Int. Endod. J., 37(2):145-50, 2004.

Linde, A. The extracellular matrix of the dental pulp and dentin. $J$. Dent. Res., 64(Spec No):523-9, 1985.

Mandarim-de-Lacerda, C. A. Stereological tools in biomedical research. An. Acad. Bras. Cienc., 75(4):469-86, 2003.

Mandarim-de-Lacerda, C. A.; Fernandes-Santos, C. \& Aguila, M. B. Image analysis and quantitative morphology. Methods Mol. Biol., 611:211-25, 2010.

Mattfeldt, T.; Mall, G.; Gharehbaghi, H. \& Möller, P. Estimation of surface area and length with the orientator. J. Microsc., 159(Pt. 3):301-17, 1990.

Murray, P. E.; About, I.; Lumley, P. J.; Franquin, J. C.; Remusat, M. \& Smith, A. J. Human odontoblast cell numbers after dental injury. J. Dent., 28(4):277-85, 2000.

Murray, P. E.; About, I.; Franquin, J. C.; Remusat, M. \& Smith, A. 
J. Restorative pulpal and repair responses. J. Am. Dent. Assoc., 132(4):482-91, 2001.

Murray, P. E.; Stanley, H. R.; Matthews, J. B.; Sloan, A. J. \& Smith, A. J. Age-related odontometric changes of human teeth. Oral Surg. Oral Med. Oral Pathol. Oral Radiol. Endod., 93(4):47482, 2002.

Pashley, D. H.; Walton, R. E. \& Slavkin, H. C. In: Ingle, J. I. \& Bakland, L. K. (eds.). Endodontics. $5^{\text {th }}$ edn. London, BC Decker, 2002. pp.25-61.

Salgado, A. G.; Vásquez, B. \& del Sol, M. Stereology of the human thyroid gland. Int. J. Morphol., 29(2):562-7, 2011.

Santamaria, M. Jr.; Milagres, D.; Stuani, A. S.; Stuani, M. B. \& Ruellas, A. C. Initial changes in pulpal microvasculature during orthodontic tooth movement: a stereological study. Eur. J. Orthod., 28(3):217-20, 2006.

Sentut, T.; Kirzioglu, Z.; Gökcimen, A.; Aslan, H. \& Erdogan, Y. Quantitative analysis of odontoblast cells in fluorotic and nonfluorotic primary tooth pulp. Turk. J. Med. Sci., 42(2):3517, 2012.

Trowbridge, H.; Kim, S. \& Suda, H. Estructura y funciones del complejo dentino-pulpar. En: Cohen, S. \& Burns, R. (Eds.). Vías de la Pulpa. 8a ed. Madrid, Mosby, 2002.

Trujillo, E.; Vásquez, B. \& del Sol, M. Stereologic Characteristics of the Liver of Rats (Rattus norvergicus) Submitted to Ovariectomy-induced Menopause. Int. J. Morphol., 29(4):1470-8, 2011.

Tziafas, D. Mechanisms controlling secondary initiation of dentinogenesis: a review. Int. Endod. J., 27(2):61-74, 1994.

Tziafas, D. Dentinogenic potential of the dental pulp: facts and hypotheses. Endod. Top., 17(1):42-64, 2010.

Vavpotic, M.; Turk, T.; Martincic, D. S. \& Balazic, J. Characteristics of the number of odontoblasts in human dental pulp postmortem. Forensic Sci. Int., 193(1-3):122-6, 2009.

Vermelin, L.; Ayanoglou, C.; Septier, D.; Carreau, J. P.; BissilaMapahou, P. \& Goldberg, M. Effects of essential fatty acid deficiency on rat molar pulp cells. Eur. J. Oral Sci., 103(4):21924, 1995.

Vit salariu, A.; Caruntu, I. D. \& Bolintineanu, S. Morphological changes in dental pulp after the teeth preparation procedure. Rom. J. Morphol. Embryol., 46(2):131-6, 2005.

Warfvinge, J. Morphometric analysis of teeth with inflamed pulp. J. Dent. Res., 66(1):78-83, 1987.
Dirección para Correspondencia:

Erick Gonzalo Trujillo Hernández

Departamento de Ciencias Básicas y Biomédicas

Morfología

Universidad de Talca

Lircay s/n,

Talca

CHILE

Email: etrujillo@utalca.cl

Recibido: 24-09-2013

Aceptado: 17-12-2013 